\title{
MODELAGEM E SIMULAÇÃO DE UM REATOR DE DESLIGNIFICAÇÃO COM OXIGÊNIO
}

\author{
Jéssica da Luz Maeiski1, Ivo Neitzel2*, Diego do Mato Lara3 \\ 1 - Faculdade de Telêmaco Borba - FATEB - PR \\ 2 - Faculdade de Telêmaco Borba-FATEB - PR, ivo.neitzel@fatebtb.edu.br \\ 3 - Faculdade de Telêmaco Borba - FATEB - PR
}

\begin{abstract}
A otimização dos processos industriais por meio da simulação é uma alternativa interessante, pois possibilita um controle maior das condições operacionais do processo. Assim o objetivo desse trabalho é modelar e simular um reator de deslignificação com oxigênio, visando sua otimização. Foi avaliada a influência da fluidodinâmica do reator na simulação, o que possibilitou determinar que o escoamento ao longo do reator é não ideal, e que existem regiões no reator com a presença de canal preferencial e de zonas estagnantes o que afeta diretamente a eficiência da deslignificação com oxigênio. As simulações foram realizadas em 2D e 3D. Na simulação $2 \mathrm{D}$ verificou-se a existência de três zonas principais dentro do reator, que mostraram uma recirculação maior nas partes mais inferiores, próximas ao distribuidor, já no comprimento do reator tem-se uma corrente ascendente sem mistura significativa, e zonas de recirculação no topo. Na simulação 3D pode-se analisar uma melhoria na recirculação ao longo do reator, isso é devido aos orifícios que foram adicionados para a distribuição da polpa, também houve melhoria no coeficiente de viscosidade não sendo observado caminho preferencial próximo a parede do reator como na simulação 2D.

Palavras-chave: otimização de processos; modelagem; influência da fluidodinâmica.
\end{abstract}

\section{INTRODUÇÃO}

O processo de deslignificação com oxigênio tem se tornado extremamente importante para as tecnologias de branqueamento de celulose no século 21 . O uso de um estágio com oxigênio promove grande economia com produtos químicos e reduz os impactos ambientais causados pelas cargas poluentes dos efluentes da planta de branqueamento. Outra vantagem é que o filtrado residual desta etapa é compatível com o licor negro do processo Kraft, o que permite que o ciclo de recuperação seja mais eficiente na recuperação energética e química [5].

A deslignificação com oxigênio é uma tecnologia estabelecida mundialmente e uma operação comum nas fábricas de celulose que utilizam o cozimento Kraft. As razões para essa utilização estão embasadas no contexto ambiental (redução de carga orgânica ao efluente), bem como no contexto econômico de redução de químicos no branqueamento [1].

Alguns modelos da cinética de deslignificação com oxigênio foram criados para melhorar a seletividade nesta etapa, sendo possível alterar os reagentes deste processo assim como manipular as variáveis de controle. Os modelos criados ajudam na otimização e simulação industrial, melhorando a qualidade do produto bem como a viabilidade econômica [4].

Neste contexto o objetivo do trabalho é analisar alternativas para otimização do processo de deslignificação com oxigênio, por meio de simulação, tanto no aspecto de condições de operação, como no contexto da fluidodinâmica do reator.

\section{METODOLOGIA}

Para o estudo do reator de deslignificação com oxigênio inicialmente foi realizada uma investigação acerca da fluidodinâmica. Através das simulações em fluidodinâmica computacional, foram obtidas informações a respeito dos padrões de escoamento do reator. Em seguida foi realizado uma pesquisa sobre os modelos cinéticos que descrevem o reator de deslignificação com oxigênio. $\mathrm{O}$ modelo cinético escolhido foi implementado. A partir do modelo foi possível investigar o seu comportamento e seu impacto sobre o processo reativo. Por fim um modelo foi escolhido para avaliar o impacto da viscosidade na etapa de deslignificação com oxigênio. 


\section{CARACTERÍSTICAS DO REATOR SIMULADO}

O reator consiste de um vaso pressurizado de fluxo ascendente com um distribuidor de polpa no fundo e descarregador no topo. A simulação foi realizada em $2 \mathrm{D}$ e $3 \mathrm{D}$. O que muda na geometria 3D é que na parte inferior do reator foi adicionado quatro orifícios que fazem a distribuição da polpa na entrada do reator.

\section{FLUIDODINÂMICA DO REATOR DE DESLIGNIFICAÇÃO}

O reator de deslignificação com oxigênio foi avaliado e teve seu desempenho analisado através, primeiramente, de simulações relacionadas a fluidodinâmica do reator analisando o tempo de permanência.

Para a determinação do tempo de permanência foram lançadas 100 partículas na parte inferior do reator onde está localizado o orifício de entrada da polpa, sendo possível observar a trajetória destas partículas ao longo tempo e o tempo de permanência de cada uma delas.

\section{ESCOLHA DO MODELO CINÉTICO}

Considerando a forte visão empírica envolvida na seleção do modelo foi realizado a busca de um modelo simplificado que não imponha um esforço computacional elevado ao simulador. Os parâmetros do modelo citados na literatura apresentam ordens de grandeza e valores bastante distintos, sugerindo uma forte dependência de todas as matérias prima envolvidas e das condições operacionais.

O modelo cinético escolhido para realizar a simulação foi o modelo de Jafari (2015), que é descrito da seguinte forma [2]:

$$
\begin{aligned}
& -\frac{d L}{d t}=k_{1} \cdot\left[O H^{-}\right]^{m} \cdot\left[P_{O 2}\right]^{n} \cdot L^{q} \\
& k_{1}=A_{q} \cdot \exp \left(\frac{-E_{A}}{R T}\right)
\end{aligned}
$$

O modelo de Jafari (2015), foi simplificado e propõem-se aqui um modelo com a forma:

$$
\frac{d\left[\tilde{L}-\tilde{L}_{F}\right]}{d t}=-M_{L}^{q-1} \cdot k \cdot\left[\hat{C}_{\mathrm{NaOH}}\right]_{0}^{n} \cdot\left[p O_{2}\right]^{m} \cdot\left[\tilde{L}-\tilde{L}_{F}\right]^{q}
$$

isto é,

$$
\begin{aligned}
& \frac{d[\tilde{\lambda}]}{d t}=-\underbrace{\overbrace{M_{\mathrm{NaOH}}^{n} \cdot M_{L}^{q-1}}^{k_{M L}} \cdot k}_{k_{\text {eff }}} \cdot\left[\tilde{C}_{\mathrm{NaOH}}\right]_{0}^{n} \cdot\left[p O_{2}\right]^{m} \cdot[\tilde{\lambda}]^{q} \quad \tilde{\lambda}=\tilde{L}-\tilde{L}_{F} \\
& \frac{d[\tilde{\lambda}]}{d t}=-k_{\text {eff }} \cdot[\tilde{\lambda}]^{q}, q>0
\end{aligned}
$$


$\left[\tilde{C}_{\mathrm{NaOH}}\right]_{0}^{n}$ é a concentração mássica de $\mathrm{NaOH}$ correspondente à carga inicial de $\mathrm{NaOH}$. Desta forma o modelo permite solução analítica fechada convergindo para o valor $[\lambda]=0$, aliás, $\tilde{L}=\tilde{L}_{F}$, que corresponde ao $\mathrm{K}_{\text {Final }}=\mathrm{K}_{\text {entra }}-\Delta \mathrm{K}$ calculado considerando o consumo total de $\left[\hat{C}_{\mathrm{NaOH}}\right]_{0}^{n}$.

\section{PERDA DE VISCOSIDADE DA POLPA}

Para a simulação da degradação dos carboidratos foi utilizado o modelo proposto por Susilo (2002), onde a perda de viscosidade é utilizada para descrever a extensão da degradação dos carboidratos [3].

A equação cinética utilizada por Susilo (2002) é a seguinte [3]:

$$
\frac{d m_{n}}{d t}=k_{c} \cdot\left[O_{2}\right]^{m} \cdot\left[O H^{-}\right]^{n} \cdot m_{n}^{q}
$$

$m_{n}=\mathrm{N}^{\circ}$ moléculas de celulose, mol/tonelada de polpa.

$k_{c}=A \cdot \exp \left(\frac{-E_{A}}{R T}\right)$

onde A é o fator de frequência, $E_{A}$ é a energia de ativação de Arrhenius (kJ/mol), R é a constante universal dos gases (kJ/mol.K) e T é a temperatura (K) [3].

\section{RESULTADOS E DISCUSSÃO}

Para a análise fluidodinâmica do reator foram utilizadas duas formas de representação do escoamento para melhor entendimento: na primeira foram lançadas partículas na entrada do reator para determinação do tempo de permanência destas ao longo do reator, sendo possível determinar se o escoamento é ideal ou não ideal. Em seguida são mostradas linhas de corrente ao longo do reator evidenciando os locais de maior recirculação e canais preferenciais. As linhas de corrente são complementadas pelos vetores relacionados à velocidade local, indicando o sentido de escoamento da polpa no interior do reator.

$\mathrm{Na}$ figura 1 pode ser visto a trajetória das partículas ao logo do tempo no reator.

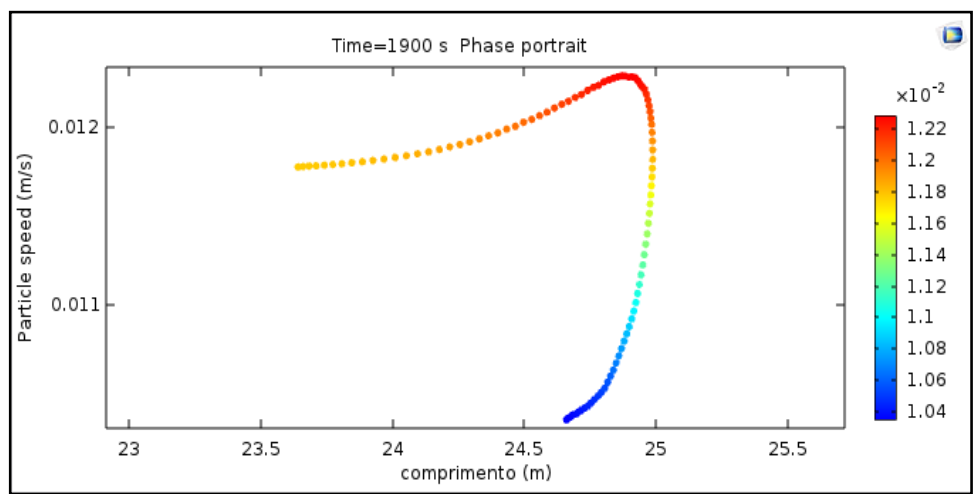

Fig 1 - Trajetória das partículas ao longo do reator.

A partir do resultado obtido na figura 1 é possível observar que a trajetória de cada partícula dentro do reator tem velocidade e posição diferente. Ao serem lançadas as partículas algumas percorrem o reator com uma velocidade maior do que as outras. Este comportamento da 
trajetória das partículas já era esperado pois elementos de fluido percorrem diferentes caminhos no reator e podem gastar tempos diferentes ao percorrerem todo o volume de controle.

A figura 2 mostra o tempo de permanência de cada partícula dentro do reator.

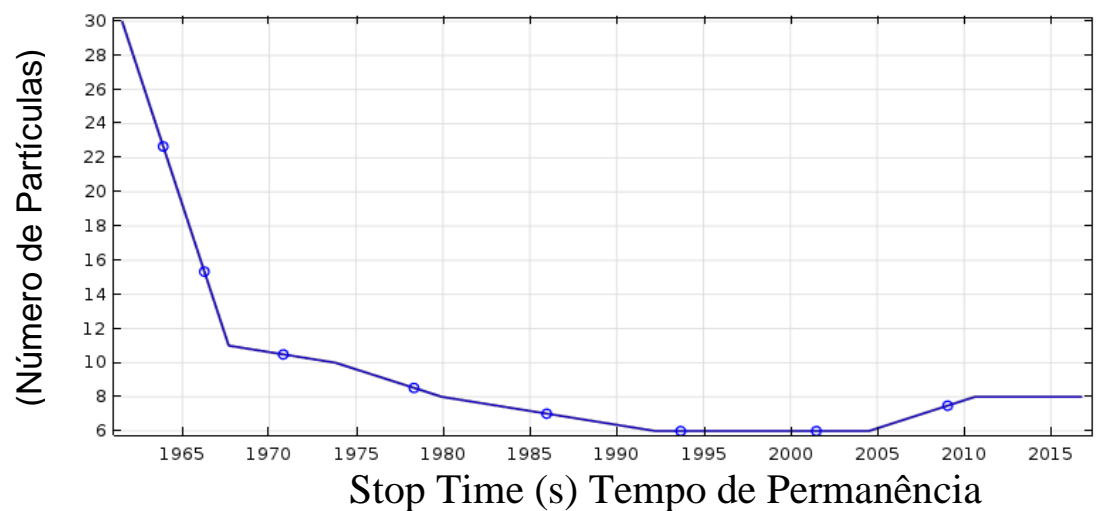

Fig 2 - Tempo de permanência das partículas no reator.

A partir do resultado nota-se que cada partícula se movimenta de forma diferente, algumas permanecem mais tempo no reator e outras menos tempo, com isso é possível caracterizar que o escoamento é não ideal pois cada partícula percorre o reator em tempos e velocidades diferentes. Esse comportamento afeta diretamente a reação química pois algumas moléculas vão ter um tempo maior de reação e outras menor, ou seja, vão existir regiões onde a reação não irá acontecer completamente causando perda de eficiência na etapa de deslignificação com oxigênio.

Esses desvios de idealidade causam no reator a formação de canais preferenciais, pelo reciclo de fluido e pelo aparecimento de regiões estagnantes no reator, isso pode ser observado nas figuras 3 e 4:

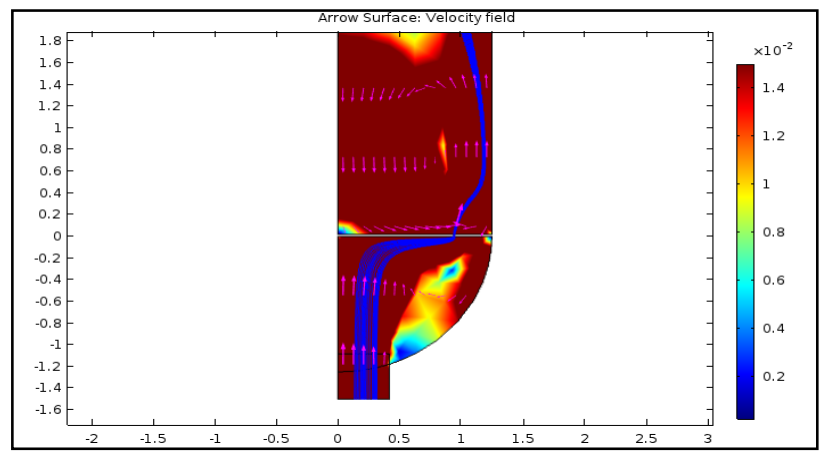

Fig 3 - Padrão de escoamento de entrada do reator.

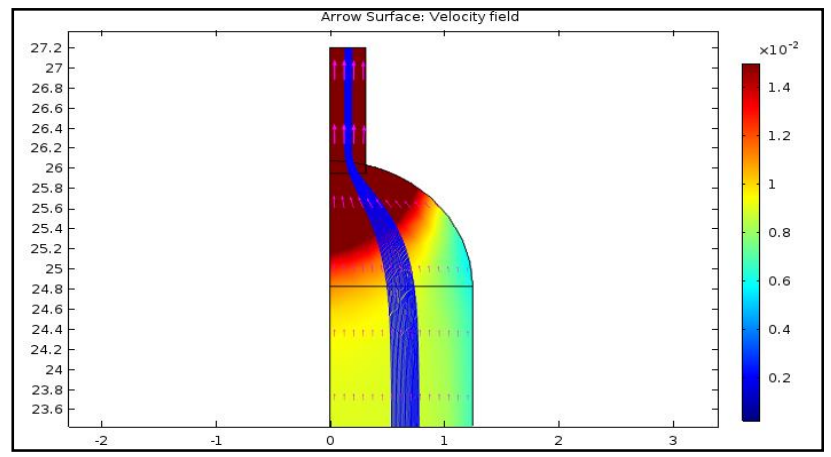

Fig 4 - Padrão de escoamento de saída do reator. 
A simulação realizada mostra a presença de canal preferencial no reator e de zonas estagnantes, trazendo muitos problemas relacionados ao escoamento da polpa, um deles é a falta de recirculação o que mostra que a mistura é ineficiente e isso é um grande problema para a operação do reator pois uma mistura ineficiente da polpa celulósica pode levar a um alto consumo de produtos químicos, baixa alvura e degradação da polpa.

As linhas de corrente mostram uma recirculação maior nas partes mais inferiores, próximas ao distribuidor. No comprimento do reator tem-se uma corrente ascendente sem mistura significativa, e por fim, zonas de recirculação no topo.

Uma forma de melhorar a recirculação no reator seria inserir mais orifícios para fazer a distribuição de entrada da polpa, isso faria com que houvesse uma mistura maior e diminuiria as regiões estagnadas e consequentemente os caminhos preferenciais. Esse comportamento pode ser observado nas simulações 3D apresentadas a seguir.

A viscosidade também é um fator que dificulta o escoamento do meio reacional, sendo este efeito mais crítico na região próxima da parede. Com os resultados obtidos é possível ver o forte impacto da viscosidade sobre a reação cinética, esse comportamento afeta e modifica a equação de transporte. Por isso a viscosidade limita a seletividade da etapa de deslignificação sendo um parâmetro de grande importância para o controle da qualidade da polpa.

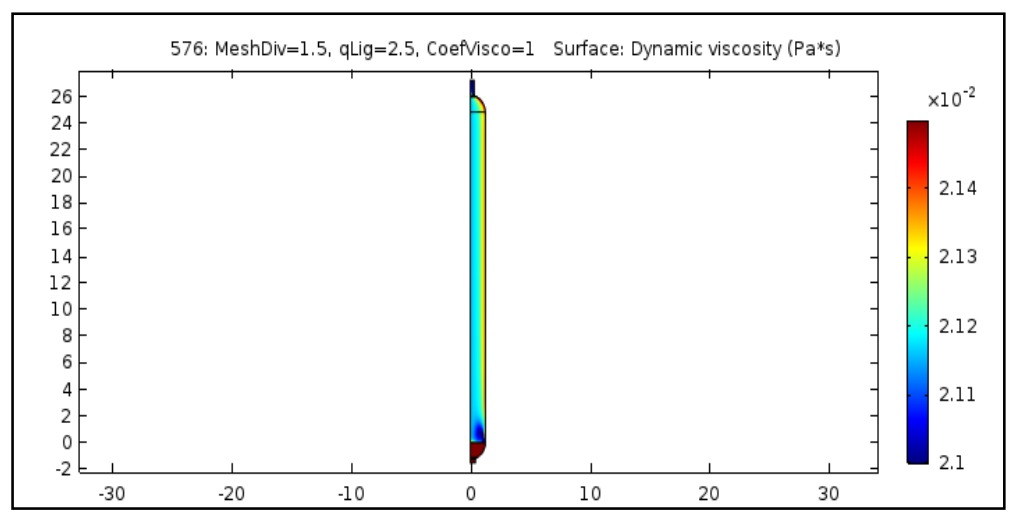

Fig 5 - Análise do coeficiente de viscosidade ao longo do reator.

Sendo a viscosidade um fator que dificulta o escoamento, pode-se perceber que na região próximo ao centro do reator a viscosidade é menor do que nas paredes, esse é um problema que afeta diretamente o perfil de velocidade dentro do reator, influenciando assim, o tempo de permanência das partículas ao longo do mesmo.

\section{SIMULAÇÃO DO MODELO CINÉTICO}

Para a simulação do modelo cinético foram estimados parâmetros de operação do reator para que representassem a etapa de deslignificação com oxigênio. As variáveis utilizadas na entrada do modelo são: número kappa na entrada do reator, pressão do oxigênio, vazão de álcali, temperatura de reação, tempo de retenção, consistência média da polpa de $10 \%$ e viscosidade. $\mathrm{Na}$ figura 6 pode-se verificar o kappa médio encontrado na saída do reator. 


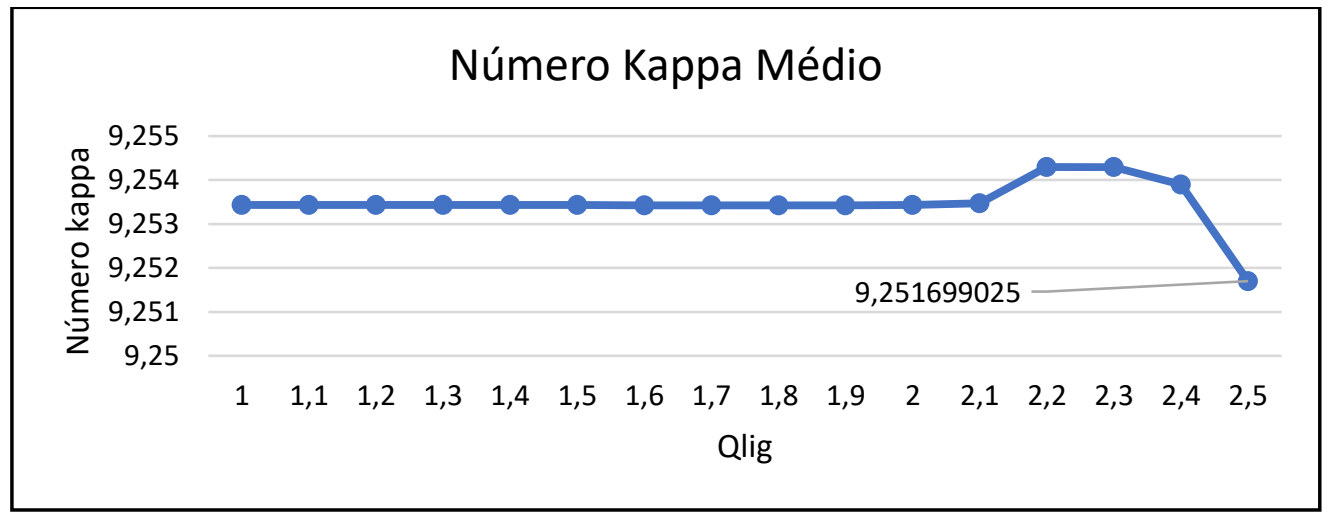

Fig 6 - Número kappa na saída do reator.

Para obtenção do número kappa na saída do reator foi especificado o kappa médio de entrada de 18,79. Na figura 6 é observado que ocorre uma convergência para um valor do número kappa médio na saída do reator de aproximadamente 9,2516, os valores encontrados na simulação 2D estão dentro do esperado, pois a redução do número kappa deve ser aproximadamente 50\% do kappa de entrada e isso é observado pois o kappa de saída teve uma diferença de 9,54 em relação ao kappa de entrada. É preciso considerar ainda, que o valor inicial do número kappa de entrada é um valor médio e que não expressa, necessariamente, a realidade modelada.

\section{SIMULAÇÃO EM 3D}

Foram realizadas simulações 3D para avaliar o comportamento do modelo implementado e da geometria do reator.

A partir do modelo de Jafari (2015) que foi simplificado pode-se prever o número kappa ao longo do reator.

Na figura 7 é mostrado o número kappa ao longo do reator.

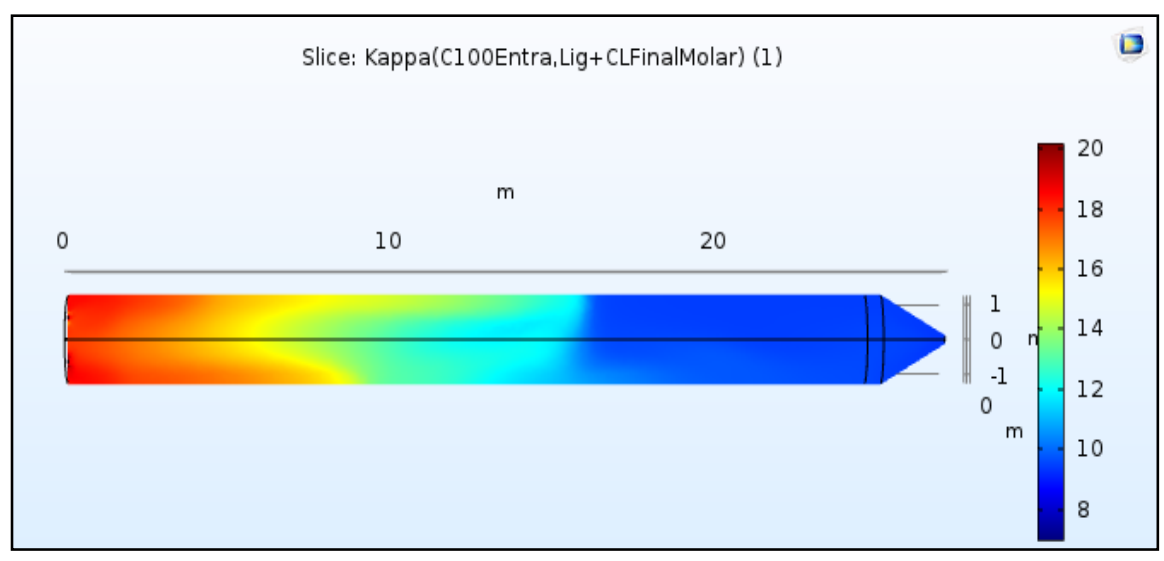

Fig 7 - Análise do número kappa.

Pode-se notar que no início da simulação o kappa está a aproximadamente 18.79 e no decorrer do tempo ao longo do reator o kappa vai diminuindo e converge para aproximadamente 9.49 obtendo uma diferença de 9.3 do kappa inicial. Comparando o resultado obtido na simulação 2D com a 3D o número kappa encontrado na simulação 3D também está dentro das especificações, pois a redução do número kappa deve ser aproximadamente $50 \%$ do kappa de 
entrada e esse comportamento pode observado na simulação 3D, validando assim o modelo simplificado e a simulação.

No modelo implementado por Jafari (2015) é preciso ter conhecimento da queda de álcali dentro do reator, já com o novo modelo proposto é necessário apenas conhecer a carga inicial de hidróxido de sódio, sendo possível determinar o kappa máximo e kappa mínimo em função da carga de álcali adicionada.

O número kappa está em função da carga de álcali, se a carga for grande e o reator suficientemente comprido o kappa tem uma queda rápida, já se o reator for pequeno isso não acontece.

O modelo Jafari (2015) que foi simplificado é mais simples e perfeitamente coerente e justificado com a concepção que se tem da reação.

Na figura 8 é apresentado a simulação do perfil de velocidade dentro do reator.

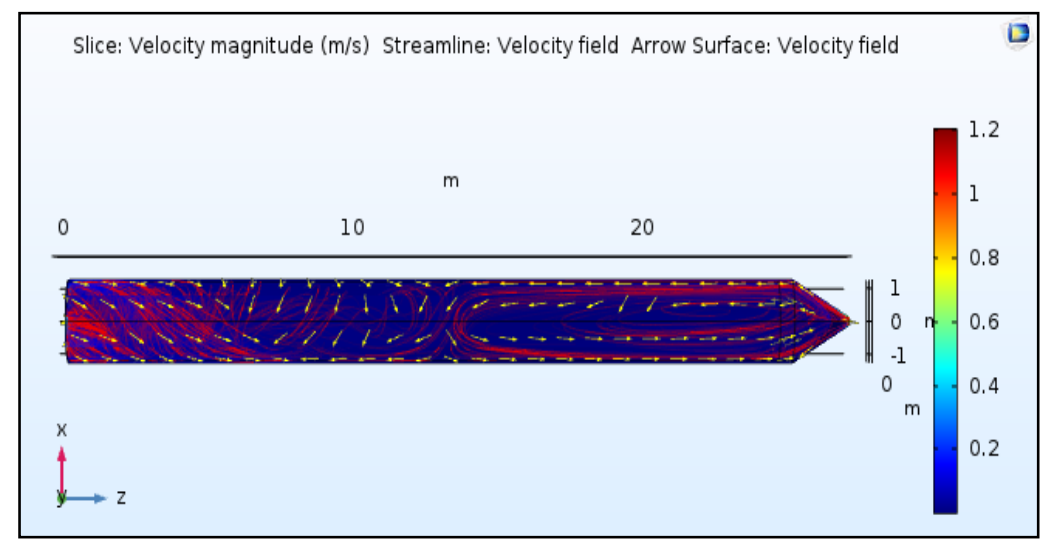

Figura 8 - Perfil de velocidade.

Com os resultados obtidos pela simulação 3D a recirculação melhorou se comparado com simulação $2 \mathrm{D}$, as linhas de corrente mostram uma maior recirculação ao longo do reator diminuindo assim os caminhos preferenciais e consequentemente a eficiência da etapa, os vetores indicam o sentido do escoamento da polpa. A velocidade é um pouco maior na entrada da polpa e isso é devido ao adicionar mais orifícios de entrada, mas ao longo do reator se mantem praticamente constante.

O coeficiente de viscosidade também foi um parâmetro que obteve uma melhora com a simulação 3D, na figura 9 é perceptível que a viscosidade no início é um pouco maior e no decorrer do comprimento do reator diminui, não foi observado caminho preferencial nas paredes do reator como na simulação 2D.

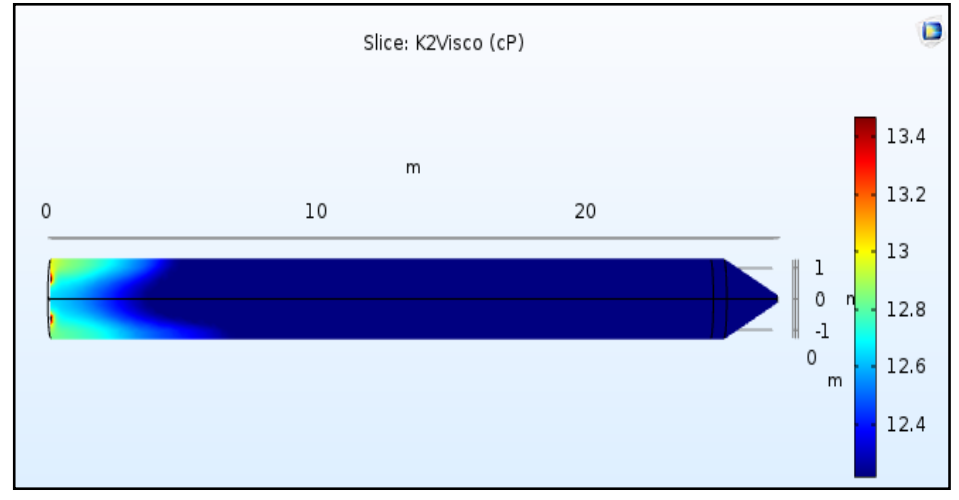

Fig 9 - Análise do comportamento da viscosidade ao longo do reator. 


\section{CONCLUSÃO}

O tempo de permanência das partículas no reator mostrou que o escoamento é não ideal o que causa problemas na eficiência do reator, como por exemplo, caminhos preferenciais e regiões estagnadas.

$\mathrm{Na}$ simulação 2D verificou-se a existência de três zonas principais dentro do reator, que mostraram uma recirculação maior nas partes mais inferiores, próximas ao distribuidor, já no comprimento do reator tem-se uma corrente ascendente sem mistura significativa, e zonas de recirculação no topo.

$\mathrm{Na}$ simulação 3D pode-se analisar uma melhoria na recirculação ao longo do reator, isso é devido aos orifícios que foram adicionados para a distribuição da polpa, também houve melhoria no coeficiente de viscosidade não sendo observado caminho preferencial próximo a parede do reator como na simulação $2 \mathrm{D}$, apesar das limitações a simulação 3D se mostrou eficiente.

O modelo cinético implementado é empírico e limitado pela falta de valores reais do processo de deslignificação com oxigênio, para obter uma maior confiabilidade ao modelo deve ser realizado um estudo buscando valores reais do processo de deslignificação com oxigênio. Dadas as limitações do modelo cinético o mesmo se mostrou eficiente para a simulação realizada.

\section{REFERÊNCIAS}

1. COLODETTE, J.L.; GOMES, F.J.B. Branqueamento da polpa celulósica: da produção de polpa marrom ao produto acabado, Viçosa, MG, p.816, 2015.

2. JAFARI, Vahid et al. Delignification and cellulose degradation kinetics models for high lignin content softwood Kraft pulp during flow-through oxygen delignification. Cellulose, v. 22, n. 3, p. 2055-2066, 2015.

3. SUSILO, Jacky. Modelling oxygen delignification in pulp processing operations. 2002. Tese de Doutorado. University of British Columbia.

4. VIANNA, Viridiane. Modelagem e simulação do processo industrial de deslignificação com oxigênio e de branqueamento ECF de polpa celulósica. 2009.

5. VIOLETTE, Steven M. Oxygen delignification kinetics and selectivity improvement. 2003. 